Universidad de Lima

Facultad de Psicología

Carrera de Psicología

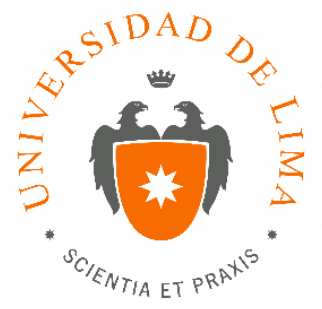

\title{
SELECCIÓN DE PERSONAL Y DESARROLLO DE INDICADORES
}

Trabajo de suficiencia profesional para optar el título profesional de Licenciado en Psicología

\section{Camila Elena Piedra Palacios}

Código 20110974

Lima - Perú

Febrero de 2019 


\section{SELECCIÓN DE PERSONAL Y DESARROLLO DE INDICADORES}




\section{TABLA DE CONTENIDO}

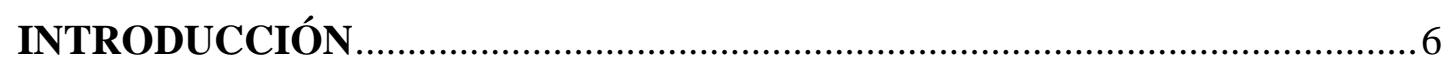

CAPÍTULO I: IDENTIFICACIÓN DEL PROBLEMA .....................................

CAPÍTULO II: DESCRIPCIÓN DE LAS ACTIVIDADES REALIZADAS ...... 10

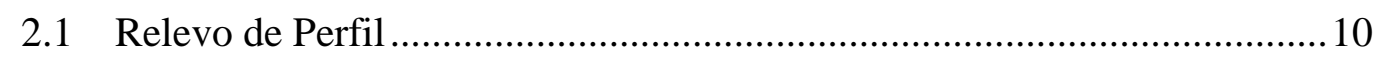

2.2 Reclutamiento y filtros preliminares …................................................ 11

2.3 Entrevistas y Dinámicas grupales ....................................................... 12

2.4 Elaboración de Informes finales............................................................ 12

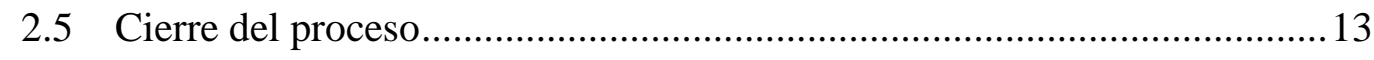

CAPÍTULO III: RESULTADOS DE LA INTERVENCIÓN ..................... 15

2.1 Selección de Personal............................................................................ 15

2.2 Implementación de indicadores ...................................................... 16

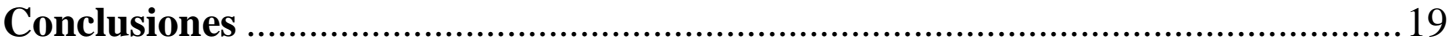

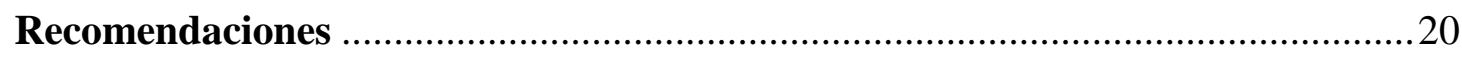

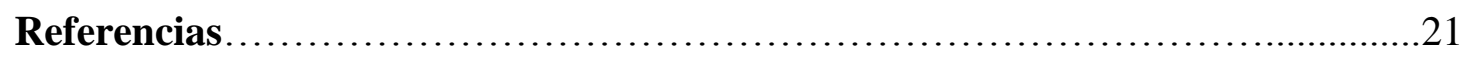




\section{ÍNDICE DE APÉNDICES}

Apéndice 1: Formato de relevo de perfil

No se encuentran elementos de tabla de ilustraciones.

Apéndice 3: Cuadro de Indicadores resumido. 


\section{INTRODUCCIÓN}

El presente trabajo tiene por finalidad exponer la experiencia obtenida en mi formación laboral dentro del área de Reclutamiento, Selección y Head Hunting en una consultora de selección, así como comentar mi participación dentro del proyecto de elaboración de indicadores para dicha consultora. El trabajo expondrá tanto la experiencia práctica como el sustento teórico correspondiente a cada tema.

Para las organizaciones resulta de suma importancia contar con la persona correcta en el puesto correcto, por lo que la selección de personal se posiciona como uno de los procesos más importantes dentro del área de RRHH. Éste, es muchas veces el primer contacto de un potencial colaborador con la empresa, y es en éste, en donde se debe buscar la idoneidad de la persona seleccionada para el puesto de trabajo (Equipo Vértice, 2018). El tercerizar este proceso puede traer beneficios en términos de ahorro de tiempo y costos por parte del área de RRHH de la empresa, siempre que sea llevado a cabo de manera ordenada y eficiente.

Por otro lado, enfocándonos en la consultora en sí, con el fin de poder medir y hacer seguimiento al rendimiento de los colaboradores de la empresa, se deben tener indicadores de productividad y efectividad. Por esto, se desarrolló un proyecto de elaboración de indicadores para la consultora, planteando mediciones relativas a la productividad diaria y efectividad en temas de tiempo e idoneidad del trabajo realizado. El uso de dichos indicadores brindará mayor información sobre los resultados del negocio, permitiendo así una toma de decisiones basadas en datos empíricos. 


\section{CAPÍTULO I: IDENTIFICACIÓN DEL PROBLEMA}

El Grupo YYYY busca ser el principal grupo multimedia del Perú, enfocado en brindar bienestar a sus consumidores y al país (Grupo YYYY, s.f.). Es poseedor de empresas de distintos rubros como entretenimiento, prensa, educación, comercio, entre otros.

En los últimos años, debido al avance tecnológico, hay mayor accesibilidad de los lectores a la información a través de medios digitales, por lo que se registró una baja en las ventas de publicidad impresa y venta de ejemplares, disminuyendo así las ganancias netas del grupo ("YYYY: ganancia neta cayó", 2017). Es por esto, que el Grupo YYYY decidió diversificar su enfoque hacia los medios de comunicación y venta electrónicos, así, WWWW pasó a formar parte del grupo en el año 2000 como empresa subsidiaria (Grupo YYYY, s.f.).

WWWW fue concebida como una empresa especializada en negocios electrónicos de tecnología web y servicios impresos. Se encontraba compuesta por diferentes unidades de negocio y productos. Sin embargo, en el 2016, algunos de estos fueron vendidos a otras empresas o cerrados debido a sus resultados comerciales (YYYY, 2016).

XXXX, uno de los productos de dicha empresa, se encuentra compuesto por suplementos impresos y electrónicos. Cada uno de los subproductos contaba con métricas propias.

En particular, XXXX es una consultora de Recursos Humanos, que tiene como clientes principales a empresas, para las cuales lleva a cabo procesos de selección, head hunting, evaluaciones psicolaborales, evaluaciones de clima laboral, entre otros. De igual manera, trabaja con personas naturales, las cuales contratan servicios de asesorías en temas relacionados a la búsqueda de empleo.

La consultora tenía objetivos mensuales netamente monetarios, que en el 2016 llegaron a alcanzar los 85000 soles anuales. Estas metas eran calculadas en base a la producción de año 
anterior y una expectativa de crecimiento, siendo la meta anual 60000 soles en el año 2015, aumentada a 85000 en el año 2016 (E. Montalvo, comunicación personal, 05 de febrero del 2019).

Los resultados obtenidos a lo largo del 2015, 2016 y 2017 se encontraron por debajo de las metas planteadas, este déficit generaba problemas al área, ya que no brindaba los resultados esperados; así como a los colaboradores, los cuales no percibían el íntegro de sus comisiones. Dicho problema, en un mediano plazo justificaría la desarticulación de la consultora. XXXX se encontraba compuesta por 7 personas, un Jefe de Producto, 2 Analistas de Selección, 2 Asistentes de Selección y 2 Practicantes de Selección. El Jefe de Producto junto con los Analistas, eran responsables de comercializar los servicios de la consultora; a su vez, los Analistas, Asistentes y Practicantes, eran los encargados de llevar a cabo dichos servicios, distribuidos en 2 equipos de 3 personas cada uno.

En esta misma línea, los resultados monetarios de la consultora dependían de las ventas realizadas en el mes, ya que se facturaba por adelantado el 50\% o 100\% del servicio; y de los procesos cerrados dicho mes, ya que se facturaría el $50 \%$ restante. Cabe mencionar, que la estructura salarial de los Analistas y Asistentes era la siguiente: $60 \%$ salario fijo mensual, $40 \%$ variable en base a los resultados comerciales y un monto variable acorde a los kilómetros recorridos en las visitas a los clientes.

A mediados del 2017, el área pasó por una reestructuración, quedando a cargo un Key Account Manager (KAM), ingresando un Ejecutivo Comercial, y formando 3 equipos de trabajo: 2 equipos compuestos por un Analista y un Practicante, y 1 equipo compuesto por un Asistente y un Practicante. Sin embargo, la estructura salarial anterior se mantuvo, a pesar de que las funciones comerciales ahora únicamente recaían sobre el KAM y el Ejecutivo Comercial. Por otro lado, en cuanto a los servicios que ofrecía la consultora, algunos eran llevados de manera externa por asesores, como la evaluación de Clima Laboral mediante la metodología 
LEGO Serious Play; mientras que otros eran llevados a cabo internamente por los equipos, como los procesos de Selección y Head Hunting.

Dichos procesos contaban con un máximo de días para la presentación de la terna finalista de acuerdo con el tipo de proceso y algunos formatos para la presentación de informes y referencias laborales al cliente, sin embargo, no se contaban con cronogramas de trabajo, cuadros de información o formatos estandarizados enfocados en el proceso para los equipos. Esto, en ocasiones representaba problemas al trasladar una cuenta de un equipo a otro, buscar información de un proceso anterior o el manejo debido de un proceso actual; ya que la información no se encontraba debidamente registrada.

Además, no se contaban con indicadores acerca del rendimiento de los colaboradores de la consultora ni de la eficacia de los procesos. Por lo que no se llevaba un debido control acerca del avance de los mismos. Esto a su vez, dificultaba el realizar una propuesta de mejora de estructura salarial en base al rendimiento individual, ya que no se contaban con las métricas necesarias.

Es así como se presentan los principales problemas a tratar: 1. La organización de los procesos de Selección, Mixtos y Head Hunting; 2. La ausencia de indicadores del área; 3. El establecimiento de metas únicamente basadas en resultados monetarios; 4. La estructura salarial. 


\section{CAPÍTULO II: DESCRIPCIÓN DE LAS ACTIVIDADES Y TAREAS REALIZADAS}

El proceso de selección de personal busca cubrir vacantes existentes dentro de las empresas, por lo que se evalúan a potenciales candidatos obtenidos durante el proceso de reclutamiento, escogiendo mediante diversas técnicas y fases a la persona más adecuada para la posición vacante, considerando tanto las necesidades de la organización, como las características personales del individuo (Pereda, Berrocal y Alonso, 2014). El proceso de selección en la consultora se llevaba a cabo en las siguientes fases:

\subsection{Relevo de Perfil}

El proceso comenzaba una vez aprobada la cotización. Se agendaba una cita para el relevo de perfil, y en esta reunión se recolectaba información acerca de los temas más relevantes a tomar en consideración para la búsqueda de candidatos, la cual correspondería al perfil del puesto. Este documento consta de una descripción de las tareas, el contexto y los requisitos fundamentales de cada posición (Alles, 2015).

Para esta parte del proceso, se buscó bibliografía al respecto de la descripción de puestos (Pereda et al., 2014; Alles, 2015) y se habló con las consultoras que componían el área, planteando así una modificación del archivo existente. Así, se planteó un formato que contaba con las siguientes categorías (Apéndice 1):

- Información sobre la posición: Funciones, nivel jerárquico, interacción con otras áreas, propósito del puesto, compensación, horario y lugar de trabajo.

- Perfil demográfico: Género, y edad.

- Experiencia Laboral: tiempo de experiencia, rubro, áreas de experiencia.

- Perfil Académico: Nivel académico, carreras, idiomas, certificaciones adicionales.

- Competencias 
- Requisitos Adicionales

Dicho documento, una vez llenado con la información recolectada, se enviaría a la empresa cliente con el fin de obtener su aprobación.

\subsection{Reclutamiento y filtros preliminares}

Una vez aprobado el perfil, comenzaría la fase de reclutamiento, en la que se busca atraer o contactar a candidatos potenciales capaces de ocupar la vacante mediante distintas técnicas. Para esto, se deben elegir los mejores medios de convocatoria acorde a la posición y realidad del mercado (Alles, 2015; Chiavenato, 2009).

Luego de haber culminado la convocatoria de candidatos, se debe realizar una preselección. El primer paso, sería el filtro curricular, que se llevaba a cabo evaluando el potencial match de la persona con la posición en base a la información brindada por el candidato en su hoja de vida, seguido por un filtro telefónico, en el cual se valida la información del candidato, se recolecta información faltante y se brinda información sobre la posición. (Perales et al., 2014).

Para poder llevar un control adecuado del proceso y los candidatos participantes, se planteó un formato en MS Excel que permitiera registrar la información de los candidatos. Este cuadro contaba con una sección fija y una variable acorde al proceso. La primera constaba de los datos principales del candidato, información personal, experiencia académica, última experiencia laboral y expectativas salariales; mientras que la variable, constaba de preguntas claves relacionadas a la posición, como, por ejemplo, el manejo de determinados softwares.

Dicho cuadro de información sería completado en los filtros telefónicos, contando además con una columna de observaciones y status que permitiera realizar seguimiento sobre el proceso de cada uno de los candidatos. De esta manera, se contaba con una base de datos por cada proceso. 


\subsection{Entrevistas y Dinámicas Grupales}

A los candidatos considerados aptos para continuar en el proceso se les citaría para una prueba, dinámica o entrevista en las oficinas de la consultora. Las técnicas a utilizar para la evaluación de candidatos dependerían de las características de la posición a cubrir. Así, en algunos procesos se realizarían dinámicas de grupo, en las que, a través de casos hipotéticos planteados a los participantes, cada uno escoge o se le asigna un rol dentro de la resolución de dicho caso, pudiendo los moderadores y observadores evaluar distintas competencias y comportamientos (Porret, 2015). Mientras que, en otros procesos, se tomarían pruebas de conocimientos, por ejemplo, al buscar un programador Java. Los candidatos seleccionados de las dinámicas y las pruebas pasarían además una entrevista individual. Por último, en algunos procesos se realizaría únicamente la entrevista por individual. En esta, el entrevistador buscará recolectar información faltante sobre el postulante, profundizar en ciertas áreas y explorar sus competencias (Porret, 2015).

Para esta fase del proceso, se decidió elaborar una guía de evaluación para las dinámicas grupales, en las que acorde a los aspectos a evaluar se incluiría la definición del comportamiento observado y una guía de clasificación. De esta manera las observadoras podrían puntuar basándose en criterios claramente establecidos, disminuyendo la subjetividad en dicha evaluación. Esta guía variaría en base al proceso, por lo cual se comenzó a desarrollar a su vez, una base de competencias con los comportamientos acorde a cada puntuación.

\subsection{Elaboración de informes finales}

Luego de haber culminado las entrevistas con los candidatos, se les enviaban evaluaciones psicológicas. Éstas eran escogidas en base al nivel jerárquico y 
funciones de la posición. En el caso de XXXX, se contaba con una herramienta de evaluación online, así las pruebas eran realizadas de manera virtual por los candidatos. Tras haber recibido el resultado de las evaluaciones finales, y decidir quiénes compondrían la terna finalista, se procedían a elaborar los files que serían entregados al cliente. Éstos estaban conformados por un informe psicolaboral, referencias laborales y los resultados de la búsqueda de antecedentes.

Se revisó el formato de informe y se realizaron modificaciones al mismo (Apéndice 2). Así, el formato final estaba compuesto por información básica del candidato, los resultados e interpretación de las evaluaciones tomadas, las competencias observadas y recomendaciones finales. Además, se incluyó un apartado de antecedentes dentro del formato, desestimando así el informe completo de la revisión de antecedentes por un tema de protección de la información personal. Vale recalcar que el candidato firmaba una autorización para realizar dicha búsqueda.

\subsection{Resultados y cierre del proceso}

Tras haber enviado los informes, la terna finalista acudía a entrevista con el cliente final, y, luego de esto se tomaba la decisión sobre el candidato seleccionado. El proceso de contratación se encontraba a cargo de la empresa cliente, por lo que la labor de la consultora para con el cliente, acababa una vez tomada la decisión. Por otro lado, dando un cierre al proceso, se comunicaba el resultado final a los participantes que no quedaron seleccionados mediante una carta de agradecimiento por su participación.

Por otro lado, un factor esencial para comprender los resultados de un negocio, son los indicadores de su gestión, éstos representan de manera cuantitativa la realidad del mismo, 
facilitando realizar un seguimiento al progreso en el alcance de los objetivos organizacionales y la medición de la efectividad de las acciones realizadas (Villagra, 2016; Mazabel, 2015). Así, los indicadores pueden ser definidos como "datos estadísticos que reflejan los resultados de una actividad en un periodo de tiempo definido" (Mazabel, 2015, p. 23). Y, cumplirían propósitos claves como ayudar a alinear los objetivos de las empresas con las estrategias empleadas, brindar información para la toma de decisiones, evaluaciones de desempeño, poder reconocer a los talentos de la empresa, entre otros (Villagra, 2016; Mazabel, 2015).

En XXXX, el desempeño del área se medía bajo un único indicador sujeto al monto facturado del mes, en otras palabras, resultados comerciales. Por esto, se decidió realizar una propuesta de nuevos indicadores en los que los colaboradores del área pudieran ver los resultados de su productividad reflejados, y que pudieran brindar información necesaria para plantear una reestructuración salarial. Así, los indicadores planteados fueron:

- Duración del proceso: (Día de aprobación del perfil - Día de entrega de informes finales) / tiempo establecido x 100

- Efectividad del proceso de hunting: $\left(\mathrm{N}^{\circ}\right.$ de candidatos con los que se establece comunicación $/ \mathrm{N}^{\circ}$ de candidatos "hunteados") x 100

- Efectividad del proceso: ( $\mathrm{N}^{\circ}$ de procesos / $\mathrm{N}^{\circ}$ de ternas enviadas) x 100

- Asistencia: ( $\mathrm{N}^{\circ}$ de candidatos que asistieron a entrevista / $\mathrm{N}^{\circ}$ de candidatos citados) x 100

- Rotación del personal contratado (dentro del periodo de garantía): $\mathrm{N}^{\circ}$ de empleados que abandonaron la empresa o fueron despedidos en el periodo de garantía por mes

- Productividad: ( $\mathrm{N}^{\circ}$ de procesos cerrados dentro del tiempo asignado en el mes $/ \mathrm{N}^{\circ}$ de procesos que deberían cerrar en el mes) x 100

El planteamiento de dichos indicadores se realizó con tres propósitos: 1. El que los colaboradores de la empresa pudieran hacer un seguimiento de su productividad, 2. Brindar la información necesaria a la empresa acerca del desempeño de los colaboradores, y 3. Realizar una propuesta de modificación de la estructura salarial en base a resultados individuales acorde a las funciones de cada colaborador. 


\section{CAPÍTULO III: RESULTADOS DE LA INTERVENCIÓN}

\subsection{Selección de Personal}

Se presentaron los formatos elaborados a las consultoras, detallando cómo debían ser llenados y manejados durante los diversos procesos. Éstos fueron recibidos de manera positiva e implementados rápidamente en los procesos que se encontraban manejando en el momento, así como en los que estaban por comenzar.

Luego de unas semanas de haber implementado dichos formatos, se llevó a cabo una reunión general para conocer las opiniones de las consultoras y obtener feedback de los mismos de manera que se pudieran hacer correcciones en caso fuera necesario. En esta reunión, se mostró un alto grado de satisfacción con las herramientas propuestas y se plantearon algunas mejoras como la elaboración de un segundo formato de informe para posiciones operativas o procesos masivos.

La utilización de dichos formatos contribuyó a la organización interna de la consultora, ya que estandarizaron maneras de manejar los procesos y la información recolectada, asegurando a la vez que ésta se encuentre debidamente registrada; alcanzando así la meta planteada.

El proceso de reclutamiento y selección de personal provee a la empresa de los recursos humanos que necesita para el alcance de sus objetivos, es por esto que debe ser llevado a cabo de manera ordenada y sistemática. El contar con el personal adecuado para la organización permitirá que ésta mantenga o aumente su eficacia como empresa, por lo que en el proceso se debe tomar en cuenta que no siempre se busca al mejor sino al más adecuado, y para poder tomar una decisión correcta, se debe contar con la información necesaria (Chiavenato, 2017). 
En el caso de XXXX, al ser una consultora que maneja procesos de empresas externas, es importante contar con toda la información relevante acerca de la misma y de la dinámica de la posición. Ya que, si bien la decisión final es tomada por la empresa cliente, los descartes realizados para llegar a la terna finalista son realizados por la consultora, quien debe tomar en consideración no sólo las habilidades técnicas del candidato, sino también sus competencias, habilidades y características personales. Además, el manejar dichos formatos de registro de información, permitió alcanzar un mayor nivel de participación de las empresas clientes en el proceso, por ejemplo, enviando listas preliminares de los candidatos para recibir retroalimentación de los mismos.

\subsection{Implementación de indicadores}

La propuesta de indicadores se realizó primero ante la jefatura, y luego ante la Gerencia de producto. Fue recibida de manera positiva y se acordó que la jefatura sería quien esté a cargo de manejar la información necesaria para dichas métricas, información que se obtendría de las reuniones de seguimiento semanales con los diferentes equipos. Además, se acordó que los indicadores se encontrarían en un archivo compartido con el equipo de la consultora para que se pueda realizar un seguimiento individual, tanto de los procesos como del desempeño personal.

La estrategia para llegar finalmente a la implementación fue la siguiente: Se tuvo una presentación de los indicadores a las colaboradoras de XXXX. En ésta se les explicó de qué trataba cada indicador planteado, de qué manera iban a ser medidos, cuáles eran los resultados esperados para cada indicador y cómo se manejaría dicha información. Luego, en las reuniones de feedback semanal, la jefatura obtenía los datos necesarios para el cálculo de dichas mediciones, por ejemplo, cuántos procesos 
se han cerrado en la semana, en qué fase se encontraba cada proceso, entre otros datos. Esa información era trasladada a un cuadro de Excel por la jefatura, quien actualizaba los archivos de manera semanal para poder realizar un seguimiento debido, teniendo así una pestaña de resumen mensual y una de seguimiento semanal (Apéndice 3).

En cuanto al aporte de la implementación, los indicadores permitieron a las colaboradoras hacer un seguimiento más detallado de su propio rendimiento. Así, cada individuo podría organizarse de una mejor manera, llevando control sobre su progreso diario. En cuanto a la organización, estos datos ayudaron a que se identificaran tanto fortalezas, dando paso a la presencia de reconocimientos; como necesidades de capacitación, las cuales, tras haber sido identificadas, permitieron que se formule un plan de mejora a través de capacitaciones individuales y grupales. Por otro lado, las métricas obtenidas fueron agregadas a las presentaciones comerciales con los clientes, dando así evidencia de la efectividad de los procesos llevados a cabo, por lo que, si bien no se planteó en un inicio como uno de los objetivos, la presencia de los indicadores influyó en el área comercial de manera positiva. Tomando en cuenta el uso comercial de dichas métricas se decidió hacer seguimiento de una adicional que abarcaría el nivel de satisfacción general de los clientes para con los distintos procesos.

Luego de la obtención de dichas mediciones se lograron identificar áreas de análisis evaluando, cuando el indicador estuviera por debajo de la meta planteada, el procedimiento que se siguió y las situaciones que dieron como resultado esa métrica. Así, se pudo identificar una necesidad de capacitación y reorganización de procesos, por ejemplo, en el caso de la efectividad de la comunicación con los candidatos pasivos, se enseñaron nuevas técnicas para lograr dicho contacto sin depender de las 
redes sociales que era la principal herramienta utilizada. Mientras que, en cuanto a la duración se procesos, se elaboró un diagrama de Gantt para cada tipo de proceso, que las consultoras pudieran utilizar adecuando únicamente la fecha de inicio de cada proceso nuevo, siendo revisado en las reuniones semanales para identificar retrasos en el desarrollo de los mismos. Por otro lado, se identificó también que el tipo de proceso llevado a cabo durante el mes afectaba significativamente algunos de los indicadores, por ejemplo, los meses en que un equipo llevara procesos masivos, el indicador referente a la asistencia de los candidatos a las entrevistas programadas sufría una baja significativa. Por eso, se decidió hacer el seguimiento de los indicadores no sólo de manera individual y grupal, sino también por proceso.

En cuanto a la propuesta de reestructuración salarial en base a los resultados de algunos de los indicadores planteados, ésta fue denegada ya que, al verse la consultora sujeta a una organización más grande, sus estructuras salariales y políticas de compensaciones provendrían de dicha organización. Y, debido a la Unidad en la que se encontraba, las posiciones eran vistas como comerciales, y por ende sujetas a metas comerciales, y el cambio implicaría una reestructuración más grande que no se podría realizar. 


\section{CONCLUSIONES}

- El relevo del perfil es la fase más importante del proceso de reclutamiento y selección, ya que es la base de las decisiones que se llevarán a cabo durante el desarrollo del proceso.

- Toda consultora dedicada a reclutamiento y selección, debe mantener orden en sus procesos y formatos estandarizados, de manera que la información sea debidamente registrada.

- Al plantear indicadores, se debe tener en cuenta el objetivo de área, así como el de la empresa.

- Los indicadores facilitan el desarrollo de una cultura orientada a objetivos dentro de la empresa. 


\section{RECOMENDACIONES}

- El relevo de perfil debe estar enfocado en entender el íntegro de la posición, no únicamente sus funciones, por lo que es importante involucrar al personal directamente relacionado con el puesto de trabajo.

- La información recolectada en el relevo de perfil puede ser utilizada en otras áreas de RRHH, como capacitación, evaluación de desempeño, planes de sucesión, entre otras. Por lo que es importante mantener esa información actualizada y recolectarla de manera adecuada.

- Para llevar un control adecuado de los procesos del área, es importante que la información sea debidamente documentada, por lo que se debe contar con indicaciones y formatos orientados al registro de información relevante.

- Toda área debe contar con métricas sobre su desempeño y productividad, por lo que es importante elaborarlas y poner la información a disposición del personal que integre dicha área. 


\section{REFERENCIAS}

Alles, M. (2015). Dirección estratégica de recursos humanos: gestión por competencias $\left(3^{\mathrm{a}}\right.$ ed.). Buenos Aires: Granica.

Chiavenato, I. (2009). Gestión del talento humano (3ª ed.). México D.F.: Mc Graw Hill

Chiavenato, I. (2017). Administración de recursos humanos: el capital humano de las organizaciones (10 ${ }^{\mathrm{a}}$ ed.). México D.F.: Mc Graw Hill

YYYY: ganancia neta cayó 80\% por menores ingresos en el tercer trimestre. (octubre, 2017). Semanaeconomica.com. Recuperado de http://semanaeconomica.com/ article/sectores-y-empresas/servicios-profesionales/250800-ganancia-neta-cayo-80por-menores-ingresos-en-el-tercer-trimestre/

YYYY S.A. (9, diciembre, 2016). Memoria Anual 2016. Recuperado del sitio de Internet de la $\begin{array}{llll}\text { Bolsa de } & \text { Valores }\end{array}$ https://www.bvl.com.pe/hhii/B30241/20170306195901/MEMORIA322016.PDF

Equipo Vértice. (2018). Selección de Personal. España: Editorial Vértice

Grupo YYYY (s.f.). Manual de Inducción. Lima: Autor

Pereda Martín, S., Berrocal Berrocal, F. y Alonso García, M. (2014). Bases de psicología del trabajo para la gestión de recursos humanos. Madrid: Síntesis.

Mazabel, C. (2015). Indicadores de recursos humanos y su resultado económico en la empresa: de lo cualitativo a lo cuantitativo. Lima: Centro de Investigaciones de Recursos Humanos

Porret, M. (2010). Gestión de personas: manual para la gestión del capital humano en las organizaciones ( $4^{\mathrm{a}}$ ed.). Madrid: ESIC

Villagra, J. (2016). Indicadores de gestión: un enfoque práctico. México D.F.: CENGAGE Learning 


\section{APÉNDICES}




\section{APÉNDICE 1: FORMATO DE RELEVO DE PERFIL}

\begin{tabular}{|l|l|}
\hline NOMBRE Y GIRO DE LA EMPRESA & TIPO DE SERVICIO (Selección, Hunting, Mixto) \\
\hline & \\
\hline
\end{tabular}

\begin{tabular}{|l|l|}
\hline PUESTO & VACANTES \\
\hline & \\
\hline
\end{tabular}

PROPÓSITO DEL PUESTO

SUPERVISIÓN

\begin{tabular}{|l|l|}
\hline REPORTA A & PERSONAL A CARGO \\
\hline & \\
\hline
\end{tabular}

USUARIOS CON LOS QUE INTERACTÚA

\begin{tabular}{|c|c|}
\hline INTERNOS & EXTERNOS \\
\hline & \\
\hline
\end{tabular}

MOTIVO DE LA CONTRATACIÓN

\begin{tabular}{|l|l|l|l|l|l|}
\hline NUEVA PLAZA & LICENCIA & & REEMPLAZO & \\
\hline
\end{tabular}

\begin{tabular}{|c|c|c|}
\hline 03 MESES & 06 MESES & OTROS \\
\hline
\end{tabular}

\begin{tabular}{|c|c|c|}
\hline $\begin{array}{l}\text { DENTRO DEL } \\
\text { ÁREA }\end{array}$ & $\begin{array}{l}\text { EN OTRA } \\
\text { ÁREA }\end{array}$ & OTROS \\
\hline
\end{tabular}




\begin{tabular}{|l|l|l|}
\hline REMUNERACIÓN & COMISIONES: & $\begin{array}{l}\text { UTILIDADES: } \\
-\end{array}$ \\
\hline RBM INICIAL: & BONOS: & \\
\hline RM FINAL: & \\
\hline INFORMACIÓN ADICIONAL: & \\
\hline
\end{tabular}

\section{CONDICIONES DE TRABAJO}

LUGAR DE TRABAJO:

HORARIO:

LOCACIÓN: CAMPO ( ) OFICINA（ )

\begin{tabular}{|l|l|l|}
\hline FORMACIÓN PROFESIONAL \\
\hline TÉCNICA - PROFESIONAL & & \\
\hline UNIVERSITARIA & & \\
\hline POSTGRADO / OTROS & & \\
\hline REQUISITOS & & \\
ADICIONALES & & \\
\hline
\end{tabular}

\begin{tabular}{|c|c|c|c|}
\hline \multicolumn{4}{|c|}{ TIEMPO DE EXPERIENCIA } \\
\hline $\begin{array}{c}6 \text { MESES - } 1 \\
\text { AÑO }\end{array}$ & $1-2$ AÑOS & $2-3$ AÑOS & \\
\hline $3-4$ AÑOS & $4-5$ AÑOS & 5-MÁS & \\
\hline \multicolumn{4}{|c|}{ ÁREAS DE EXPERIENCIA } \\
\hline \multicolumn{4}{|c|}{ TIPOS DE EMPRESAS } \\
\hline
\end{tabular}

OTROS REQUISITOS

DATOS DEMOGRÁFICOS DEL POSTULANTE

SEXO

RANGO DE EDAD 


\begin{tabular}{|c|c|c|c|}
\hline \multicolumn{4}{|c|}{ COMPETENCIAS REQUERIDAS } \\
\hline COMPETENCIAS & BAJO & MEDIO & ALTO \\
\hline & & & \\
\hline & & & \\
\hline & & & \\
\hline & & & \\
\hline & & & \\
\hline & & & \\
\hline & & & \\
\hline & & & \\
\hline
\end{tabular}

\section{FUNCIONES GENERALES Y ESPECÍFICAS}

\begin{tabular}{|c|c|}
\hline IDIOMAS & \\
\hline IDIOMA & NIVEL \\
\hline- & - \\
\hline
\end{tabular}

\begin{tabular}{|c|c|}
\hline MANEJO DE HERRAMIENTAS TECNOLÓGICAS & \\
\hline SOFTWARE & \multicolumn{1}{c|}{ NIVEL / ESPECIFICACIONES } \\
\hline- & - \\
\hline
\end{tabular}

\begin{tabular}{|l|}
\hline INFORMACIÓN ADICIONAL \\
\hline RUBROS DE INTERÉS \\
\hline \\
\end{tabular}

FECHA DE HOY:

NOMBRES Y APELLIDOS DEL CLIENTE:

FIRMA DEL CLIENTE 


\section{APÉNDICE 2: INFORME PSICOLABORAL}

La información de este reporte es confidencial. El uso se restringe únicamente a las personas involucradas directamente en la selección del candidato. La información contenida en este informe es en base a los datos reportados por el candidato a XXXX, resultados de las pruebas aplicadas y la evaluación por competencias.

\section{IDENTIFICACIÓN DEL POSTULANTE}

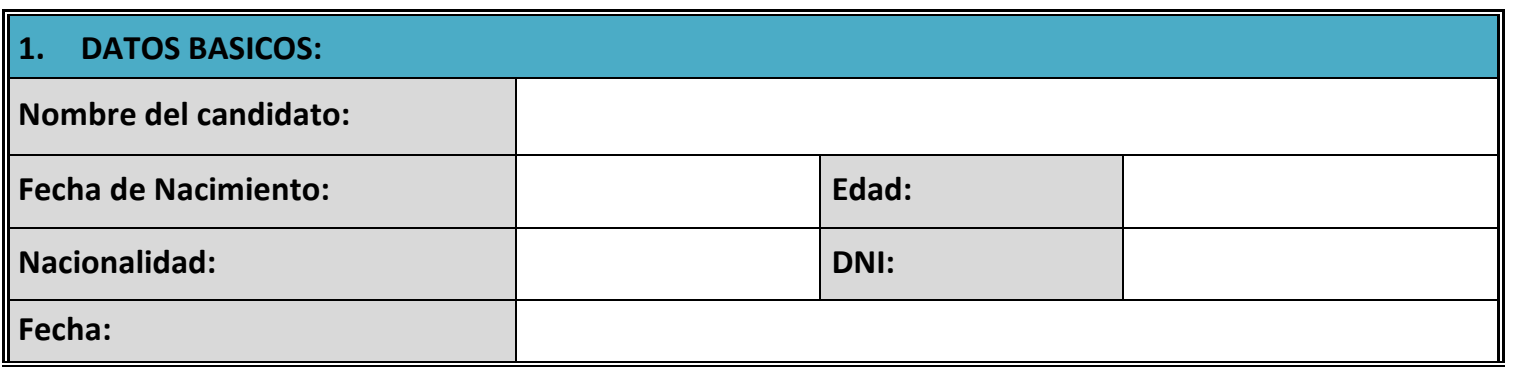

\section{PERFIL DEL POSTULANTE}

1. INTELIGENCIA COGNITIVA

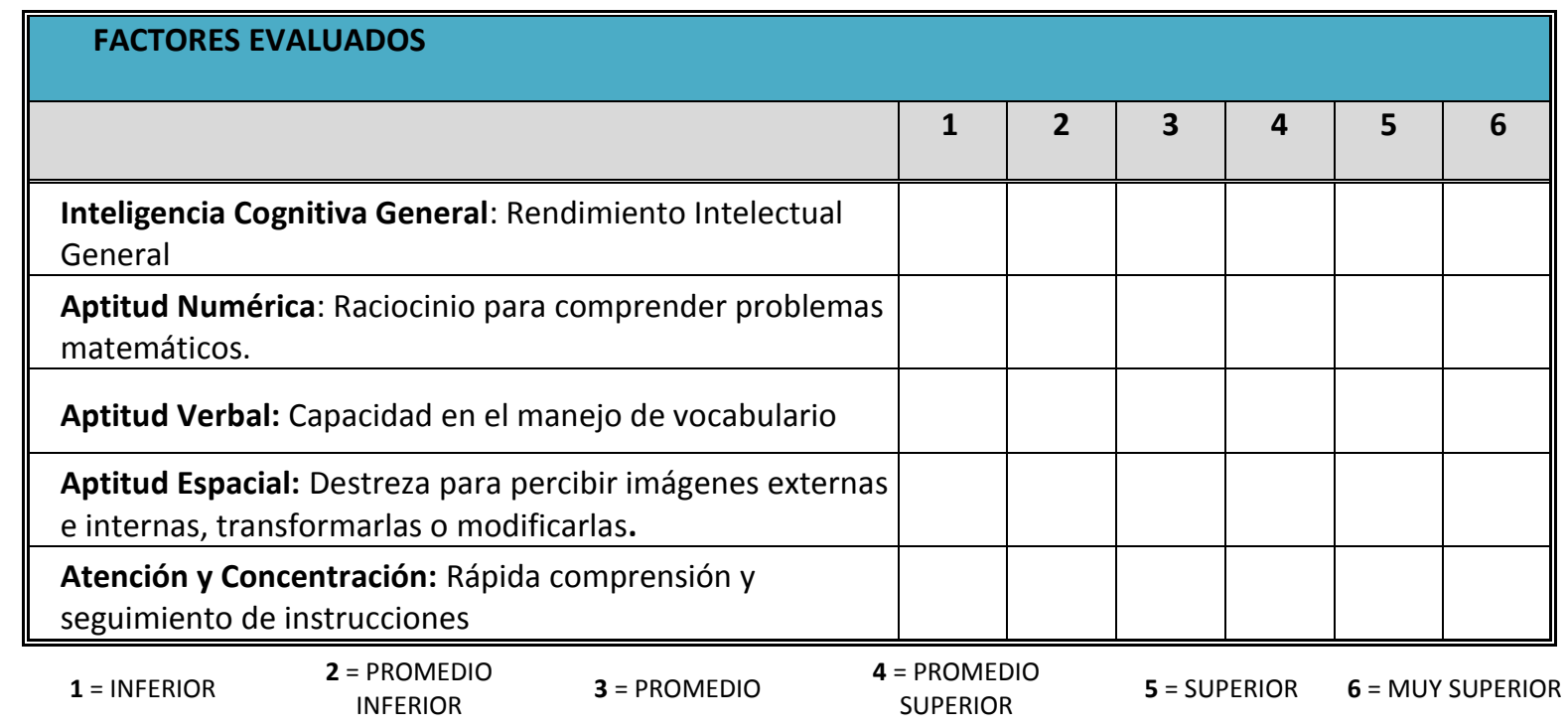

\section{RESUMEN}


2. PERFIL DE PERSONALIDAD

\begin{tabular}{|c|c|c|c|c|}
\hline & A & B & C & D \\
\hline Competencia + Descripción & & & & \\
\hline Competencia + Descripción & & & & \\
\hline Competencia + Descripción & & & & \\
\hline
\end{tabular}

A: Alto B: Bueno C: Mínimo esperado D: Insatisfactorio

IV. FORTALEZAS Y AREAS DE MEJORA

\begin{tabular}{|l|l||}
\hline \hline FORTALEZAS & OPORTUNIDADES DE MEJORA \\
\hline \hline$\bullet$ & $\bullet$ \\
\hline$\bullet$ & \\
\hline
\end{tabular}

\section{ANTECEDENTES}

\begin{tabular}{|c|c|c|}
\hline POLICIALES & JUDICIALES & PENALES \\
\hline \hline & & \\
\hline
\end{tabular}

VI. CONCLUSIONES Y DIAGNOSTICO PSICOLABORAL 


\section{APÉNDICE 3: CUADRO DE INDICADORES RESUMIDO}

\begin{tabular}{|c|c|c|}
\hline \multicolumn{3}{|c|}{ Equipo 1} \\
\hline Indicadores & $\begin{array}{l}\text { Noviembre } \\
\text { '17 } \\
\end{array}$ & $\begin{array}{l}\text { Diciembre } \\
117 \\
\end{array}$ \\
\hline Duración & $130 \%$ & $130 \%$ \\
\hline Efectividad Hunting & $52 \%$ & - \\
\hline Efectividad Proceso & $56 \%$ & $70 \%$ \\
\hline Asistencia & $97 \%$ & $80 \%$ \\
\hline Rotación & 0 & 1 \\
\hline Productividad & $60 \%$ & $83 \%$ \\
\hline Resultados Comerciales & $85 \%$ & $87 \%$ \\
\hline Satisfacción del Cliente & $85 \%$ & $80 \%$ \\
\hline
\end{tabular}

